\title{
Acentrosomal microtubule assembly in mitosis: the where, when and how
}

Sylvain Meunier ${ }^{1,2}$ and Isabelle Vernos ${ }^{1,2,3}$

${ }^{1}$ Cell and Developmental Biology Programme, Centre for Genomic Regulation (CRG), Doctor Aiguader, 88, 08003 Barcelona, Spain

2Universitat Pompeu Fabra (UPF), Doctor Aiguader, 88, 08003 Barcelona, Spain

${ }^{3}$ Institució Catalana de Recerca I Estudis Avançats (ICREA), Passeig de Lluis Companys 23, 08010 Barcelona, Spain

\section{Keywords}

Microtubules; mitosis; acentrosomal pathways; RanGTP; kinetochores; augmin. 


\begin{abstract}
In mitosis the cell assembles the bipolar spindle, a microtubule-based apparatus that segregates the duplicated chromosomes into the two daughter cells. Most animal cells enter mitosis with duplicated centrosomes that provide an active source of dynamic microtubules (MTs). However, it is now established that spindle assembly relies on the nucleation of acentrosomal MTs occurring around the chromosomes after nuclear envelope breakdown, and on pre-existing microtubules. Where chromosome-dependent MT nucleation occurs, when MT amplification takes place and how the two pathways function are still key questions that generate some controversies. Here, we review the literature to try to reconcile the data and present an integrated model accounting for acentrosomal microtubule assembly in the dividing cell.
\end{abstract}




\section{Text}

\section{Introduction}

When the cell enters mitosis, the relatively stable interphase microtubules (MT) disassemble and highly dynamic MTs organize and build the bipolar spindle, the molecular machine that drives chromosome segregation. Most animal cells enter mitosis with two centrosomes that actively nucleate dynamic MTs ${ }^{1,2}$. In addition, acentrosomal pathways trigger MT assembly in the dividing cells ${ }^{3}$. These MTs are now recognized as essential and sufficient for the assembly of a functional bipolar spindle. This is particularly obvious in cells that are naturally devoid of centrosomes (like plants cells and vertebrate oocytes for example) ${ }^{4}$, but bipolar spindles also assemble in cells experimentally manipulated to eliminate their centrosomes ${ }^{5-7}$. More strikingly, flies engineered to lack functional centrosomes were shown to develop till adulthood ${ }^{8,9}$. Altogether these data underscore the essential role of acentrosomal MT assembly pathways for cell division.

Two main mechanisms drive acentrosomal MT assembly in the dividing cells. One of them is triggered by the chromosomes (RanGTP and CPC-dependent pathways, see boxes 1 and 2) and the other drives MT amplification through a MT-dependent MT nucleation mechanism (Augmin pathway, see box 3). Although the identification of these pathways has been instrumental to explain how the spindle forms, there are currently some controversies on important questions. Where precisely chromosome-dependent MT nucleation occurs is one of them. Although this pathway can only function after nuclear envelope breakdown, it is not clear whether this is also the case for the augmin-dependent pathway. Finally, how the two acentrosomal MT nucleation pathways work and whether they are independent or not is currently unclear. 
Here we review the data that have accumulated over the years on acentrosomal MT assembly in the dividing cell. Trying to reconcile these data and some controversies, we envision that acentrosomal MT assembly occurs through a sequence of events starting with RanGTP-dependent MT nucleation in the vicinity of the chromosomes followed by MT stabilization in the kinetochore area, and MT amplification through the augmin-dependent MT nucleation pathway. Finally, we propose a mechanism for the integration of the acentrosomal MT assembly pathways in mitosis, leading to the formation of the kinetochore fibers (K-fibers) within the bipolar spindle.

\section{Chromosome-dependent MT assembly in the dividing cell}

The assembly of MTs in the proximity of the chromosomes was reported as early as in the 70 s and the 80 s by various groups, based on observations done upon MT regrowth after MT-depolymerizing drug washouts ${ }^{10-12}$ and in vitro MT nucleation assays on chromosomes 13-16. Altogether the data suggested that mitotic chromosomes could induce MT assembly in a region close to their kinetochores ${ }^{17}$.

Some reports on $\gamma$-tubulin localization at or close to the kinetochores in mitosis 18, 19 fueled the idea that kinetochores could in fact directly nucleate MTs during mitosis ${ }^{20}$. However, this also posed a problem because the nucleation of MTs at the kinetochores would inevitably result in their "reversed" polarity with their minus-end embedded at the kinetochore, and their plus-end extending away towards the spindle poles. This orientation has never been observed in animal cells ${ }^{21-23}$. It has however been shown to occur transiently in budding yeast and favor the search and capture of the chromosomes ${ }^{24}$. The mechanism in this case 
involves a mitotic MAP associated to the kinetochore and not canonical $\gamma$-tubulin dependent MT nucleation ${ }^{24}$.

Other approaches had indicated that MT assembly could be triggered by the Mphase chromatin. Experiments performed in the 80s showed that injection of phage DNA into Xenopus eggs triggered spindle assembly suggesting that the pathway could function in the absence of kinetochores ${ }^{25}$. Further support for this idea was obtained by showing that DNA coated beads promoted the assembly of bipolar spindles in Xenopus egg extracts ${ }^{26}$. Moreover, experiments in grasshopper spermatocytes showed that chromatin rather than kinetochores triggered acentrosomal MT assembly 27.

How is it possible to reconcile all these data?

A major breakthrough in our understanding of the mechanism by which chromatin induces MT assembly came with the identification of the RanGTP pathway ${ }^{28-30}$ (see box 1). This pathway is triggered by the chromatin through its associated Ran GEF, RCC1 (see box 1). Strikingly, addition of recombinant RanGTP to Xenopus M-phase egg extracts triggers the pathway in the absence of chromatin ${ }^{28}$. We now understand how RanGTP triggers MT nucleation in the Mphase cytoplasm. By releasing its direct target TPX2 from importins, RanGTP promotes the formation of a complex between TPX2 and a specific MT nucleation machinery RHAMM-NEDD1- $\gamma$ TURC promoting MT nucleation 31. These mechanistic data do not suggest any need for a predefined site at which MT nucleation occurs. Moreover, the existence of a RanGTP gradient centered on the mitotic chromatin, which has been visualized in Xenopus egg extracts and in cells, strongly favors also the idea of MT nucleation occurring in the vicinity of the chromosomes rather than at predefined specific sites such as the kinetochores. 
Kinetochores however do provide an excellent environment for MT stabilization (see box 2). Indeed, the chromosomal passenger complex (CPC) that localizes to the centromeres during mitosis has a major role in MT stabilization, as shown elegantly using the Xenopus egg extract system ${ }^{32,33}$. It is therefore possible that MTs nucleated through the RanGTP dependent pathway in the proximity of the chromosomes may get preferentially stabilized in the vicinity of the kinetochores through a phosphorylation dependent mechanism involving Aurora B within the CPC complex (see box 2) ${ }^{34}$. In this view, the observation of $\gamma$-tubulin, or of $\gamma$ TuRC components, close to or at the kinetochore in MT regrowth experiments may result from the presence of very small MTs connected to the kinetochore at their plus-end and capped by the $\gamma$ TuRC at their minus-end 18,20 .

Based on all these data, we therefore envision that MTs are nucleated through a RanGTP-dependent pathway in the cytoplasm in the close proximity of the chromosomes ${ }^{31}$ and become locally stabilized around the kinetochores through a CPC dependent mechanism before their capture and attachment at their plusend $32,33,35,36$. Once captured by the kinetochore, the growth of the MT results in its minus-end being pushed away towards the spindle pole 23,37,38. This mechanism may favor the "search and capture" of the chromosomes 39, 40 to account for the rapid attachment of all the chromosomes observed in most animal cells, and play also an important role for K-fiber assembly ${ }^{3,41}$.

\section{Augmin-dependent MT assembly - integration with the RanGTP pathway}

Recently, an additional mechanism for acentrosomal MT assembly in mitosis was identified. This pathway is dependent on the octameric augmin complex also called HAUS ${ }^{42-44}$ (see box 3 ). This complex recruits the $\gamma$ TuRC to the lattice of a 
pre-existing MT, inducing the nucleation and branching of a new MT 45,46. This amplification mechanism drives the rapid increase of the MT mass within the spindle. The augmin pathway therefore shares with the other MT assembly pathways the essential requirement for a $\gamma \mathrm{TuRC}$-dependent MT nucleation mechanism. However, in contrast to the other pathways it also requires previously assembled MTs (see box 3).

A recent study reported the coimmunoprecipitation of augmin with TPX2 (see box 1 and above), suggesting a potential direct link between the RanGTPdependent and the augmin-dependent MT assembly pathways ${ }^{45}$. Moreover, RanGTP was shown to increase the efficiency of the augmin pathway in the Xenopus egg extract system although the experimental setup also involved the addition of a large excess of TPX2 to the egg extract. These data suggest that the main function of RanGTP and TPX2 is to activate the augmin pathway. However, they can also be interpreted in a different way.

Previous work has shown that augmin depletion results in a reduction of the MT mass in the spindle in various experimental systems (mammalian cells, Drosophila cells and Xenopus egg extracts). MT assembly is therefore not completely impaired and spindles do assemble $42,43,47,48$. Indeed, Goshima et al reported that 'the Dgt complex (i.e. augmin) is dispensable for the initial nucleation of MTs around chromatin but is important for the subsequent amplification (...) of MTs" 42 . Consistently, Petry et al. also stated that: "chromatinmediated MT nucleation (...) drives the initial MT formation and appears to be augmin independent" 47. Since RanGTP induces MT nucleation in a MT-free environment 28,31 , it can provide template MTs needed for the augmin complex and the subsequent MT-dependent MT amplification. It therefore appears 
unlikely that the RanGTP and augmin dependent pathway constitute a unique pathway. Interestingly, it was recently shown that centrosomal MTs are also targets of the augmin-dependent amplification pathway in Drosophila embryos 49. This suggests that the augmin-dependent amplification mechanism can work independently of the RanGTP pathway. Nonetheless, further studies will be needed to clarify the putative functional link between TPX2 and the augmin complex, since RanGTP and TPX2 dramatically increase the efficiency of the augmin pathway ${ }^{44,45}$ (see outstanding box).

Altogether, the data favor the idea that RanGTP and the augmin MT assembly pathways are independent pathways that contribute sequentially to the assembly of acentrosomal MTs in the M-phase cytoplasm, the augmin pathway acting downstream of the RanGTP pathway for spindle formation (see our model figure 1).

\section{Concluding remarks}

Since the early description of acentrosomal MTs in mitotic cells and the subsequent findings pointing to their essential role for building a functional bipolar spindle, some basic questions are still unresolved. Here, we have reviewed data published over the last 40 years and propose a model to account for the mechanism underlying acentrosomal MT assembly in the dividing cells (Fig. 1). RanGTP triggers the initial activation of MT nucleation and stabilization around the mitotic chromosomes (Fig. 1A). These chromosomal and centrosomal MTs are then stabilized in the proximity of the kinetochores through an AuroraB/CPC dependent way (Fig. 1B). Concomitantly, chromosomal MTs act as template for augmin-dependent MT nucleation, promoting an efficient 
mechanism for MT amplification around the chromosomes (Fig. 1C). Upon MT plus-end capture by the kinetochores, the minus-ends are pushed away towards the spindle poles (Fig. 1D) 37,38, 50 .

Acentrosomal MTs are essential for the assembly and function of the bipolar spindle 41,51 . Since the chromosomal MTs become preferentially stabilized close to the kinetochores, they are at the right place to get efficiently captured, thereby promoting K-fiber assembly ${ }^{23}$. Consistently, several of the RanGTP-regulated spindle assembly factors have been found to play a role in K-fiber assembly and/or dynamics $37,41,52-54$. It is therefore tempting to propose that MT assembly around the chromosomes is essential for K-fiber formation (see outstanding box). The early observations of small MTs in the kinetochore area ${ }^{11}$ could correspond to early phases in the formation of the K-fibers constituted by large MT bundles connecting the kinetochores to the spindle poles in metaphase ${ }^{12}$. By being nucleated 31 and stabilized 41 through specific mechanisms, the chromosomal MTs may confer to the K-fiber their characteristics in terms of dynamics and organization. This may be the reason for the essential requirement of the chromosomal MTs for accurate spindle assembly and cell division. 


\section{References}

1. Wiese, C., and Zheng, Y. (2006) Microtubule nucleation: gamma-tubulin and beyond. J Cell Sci 119, 4143-4153

2. Walczak, C.E., and Heald, R. (2008) Mechanisms of mitotic spindle assembly and function. Int Rev Cytol 265, 111-158

3. Meunier, S., and Vernos, I. (2012) Microtubule assembly during mitosis from distinct origins to distinct functions? J Cell Sci 125, 2805-2814

4. Bettencourt-Dias, M. (2013) Q\&A: Who needs a centrosome? BMC Biol 11, 28

5. Khodjakov, A., et al. (2000) Centrosome-independent mitotic spindle formation in vertebrates. Curr Biol 10, 59-67

6. Mahoney, N.M., et al. (2006) Making microtubules and mitotic spindles in cells without functional centrosomes. Curr Biol 16, 564-569

7. Hinchcliffe, E.H., et al. (2001) Requirement of a centrosomal activity for cell cycle progression through G1 into S phase. Science 291, 1547-1550

8. Basto, R., et al. (2006) Flies without centrioles. Cell 125, 1375-1386

9. Megraw, T.L., et al. (2001) Zygotic development without functional mitotic centrosomes. Curr Biol 11, 116-120

10. De Brabander, M., et al. (1981) Nucleated assembly of mitotic microtubules in living PTK2 cells after release from nocodazole treatment. Cell Motil 1, 469-483

11. Witt, P.L., et al. (1980) Origin of kinetochore microtubules in Chinese hamster ovary cells. Chromosoma $81,483-505$

12. Witt, P.L., et al. (1981) Structure of kinetochore fibers: microtubule continuity and inter-microtubule bridges. Chromosoma 83, 523-540 
13. McGill, M., and Brinkley, B.R. (1975) Human chromosomes and centrioles as nucleating sites for the in vitro assembly of microtubules from bovine brain tubulin. J Cell Biol 67, 189-199

14. Pepper, D.A., and Brinkley, B.R. (1979) Microtubule initiation at kinetochores and centrosomes in lysed mitotic cells. Inhibition of site-specific nucleation by tubulin antibody. J Cell Biol 82, 585-591

15. Pepper, D.A., and Brinkley, B.R. (1980) Tubulin nucleation and assembly in mitotic cells: evidence for nucleic acids in kinetochores and centrosomes. Cell Motil 1, 1-15

16. Bergen, L.G., et al. (1980) Polarity of microtubules nucleated by centrosomes and chromosomes of Chinese hamster ovary cells in vitro. J Cell Biol 84, 151-159

17. Mitchison, T.J., and Kirschner, M.W. (1985) Properties of the kinetochore in vitro. I. Microtubule nucleation and tubulin binding. J Cell Biol 101, 755-765

18. Mishra, R.K., et al. (2010) The Nup107-160 complex and gamma-TuRC regulate microtubule polymerization at kinetochores. Nat Cell Biol 12, 164-169

19. Katayama, H., et al. (2008) Aurora kinase-A regulates kinetochore/chromatin associated microtubule assembly in human cells. Cell Cycle 7, 2691-2704

20. Torosantucci, L., et al. (2008) Localized RanGTP accumulation promotes microtubule nucleation at kinetochores in somatic mammalian cells. Mol Biol Cell $19,1873-1882$

21. Euteneuer, U., et al. (1983) Polarity of kinetochore microtubules in Chinese hamster ovary cells after recovery from a colcemid block. J Cell Biol 97, 202-208 
22. Euteneuer, U., and McIntosh, J.R. (1981) Structural polarity of kinetochore microtubules in PtK1 cells. J Cell Biol 89, 338-345

23. Rieder, C.L. (2005) Kinetochore fiber formation in animal somatic cells: dueling mechanisms come to a draw. Chromosoma 114, 310-318

24. Kitamura, E., et al. (2010) Kinetochores generate microtubules with distal plus ends: their roles and limited lifetime in mitosis. Dev Cell 18, 248-259

25. Karsenti, E., et al. (1984) Respective roles of centrosomes and chromatin in the conversion of microtubule arrays from interphase to metaphase. J Cell Biol $99,47 s-54 s$

26. Heald, R., et al. (1996) Self-organization of microtubules into bipolar spindles around artificial chromosomes in Xenopus egg extracts. Nature 382, $420-425$

27. Zhang, D., and Nicklas, R.B. (1995) The impact of chromosomes and centrosomes on spindle assembly as observed in living cells. J Cell Biol 129, $1287-1300$

28. Carazo-Salas, R.E., et al. (1999) Generation of GTP-bound Ran by RCC1 is required for chromatin-induced mitotic spindle formation. Nature 400, 178-181 29. Kalab, P., et al. (1999) The ran GTPase regulates mitotic spindle assembly. Curr Biol 9, 481-484

30. Zhang, C., et al. (1999) Ran-GTP stabilises microtubule asters and inhibits nuclear assembly in Xenopus egg extracts. J Cell Sci 112 ( Pt 14), 2453-2461

31. Scrofani, J., et al. (2015) Microtubule nucleation in mitosis by a RanGTPdependent protein complex. Curr Biol 25, 131-140 
32. Kelly, A.E., et al. (2007) Chromosomal enrichment and activation of the aurora B pathway are coupled to spatially regulate spindle assembly. Dev Cell 12, $31-43$

33. Sampath, S.C., et al. (2004) The chromosomal passenger complex is required for chromatin-induced microtubule stabilization and spindle assembly. Cell 118, 187-202

34. Tulu, U.S., et al. (2006) Molecular requirements for kinetochoreassociated microtubule formation in mammalian cells. Curr Biol 16, 536-541

35. Carmena, M., et al. (2012) The chromosomal passenger complex (CPC): from easy rider to the godfather of mitosis. Nat Rev Mol Cell Biol 13, 789-803

36. Maresca, T.J., et al. (2009) Spindle assembly in the absence of a RanGTP gradient requires localized CPC activity. Curr Biol 19, 1210-1215

37. Khodjakov, A., et al. (2003) Minus-end capture of preformed kinetochore fibers contributes to spindle morphogenesis. J Cell Biol 160, 671-683

38. Maiato, H., et al. (2004) Kinetochore-driven formation of kinetochore fibers contributes to spindle assembly during animal mitosis. J Cell Biol 167, 831840

39. Kirschner, M.W., and Mitchison, T. (1986) Microtubule dynamics. Nature 324,621

40. Wollman, R., et al. (2005) Efficient chromosome capture requires a bias in the 'search-and-capture' process during mitotic-spindle assembly. Curr Biol 15, 828-832

41. Meunier, S., and Vernos, I. (2011) K-fibre minus ends are stabilized by a RanGTP-dependent mechanism essential for functional spindle assembly. Nat Cell Biol 13, 1406-1414 
42. Goshima, G., et al. (2008) Augmin: a protein complex required for centrosome-independent microtubule generation within the spindle. J Cell Biol $181,421-429$

43. Lawo, S., et al. (2009) HAUS, the 8-subunit human Augmin complex, regulates centrosome and spindle integrity. Curr Biol 19, 816-826

44. Hsia, K.C., et al. (2014) Reconstitution of the augmin complex provides insights into its architecture and function. Nat Cell Biol 16, 852-863

45. Petry, S., et al. (2013) Branching microtubule nucleation in Xenopus egg extracts mediated by augmin and TPX2. Cell 152, 768-777

46. Kamasaki, T., et al. (2013) Augmin-dependent microtubule nucleation at microtubule walls in the spindle. J Cell Biol 202, 25-33

47. Petry, S., et al. (2011) Augmin promotes meiotic spindle formation and bipolarity in Xenopus egg extracts. Proc Natl Acad Sci U S A 108, 14473-14478 48. Uehara, R., et al. (2009) The augmin complex plays a critical role in spindle microtubule generation for mitotic progression and cytokinesis in human cells. Proc Natl Acad Sci U S A 106, 6998-7003

49. Hayward, D., et al. (2014) Synergy between multiple microtubulegenerating pathways confers robustness to centrosome-driven mitotic spindle formation. Dev Cell 28, 81-93

50. Lecland, N., and Luders, J. (2014) The dynamics of microtubule minus ends in the human mitotic spindle. Nat Cell Biol 16, 770-778

51. Gruss, 0.J., et al. (2002) Chromosome-induced microtubule assembly mediated by TPX2 is required for spindle formation in HeLa cells. Nat Cell Biol 4, 871-879 
52. Koffa, M.D., et al. (2006) HURP is part of a Ran-dependent complex involved in spindle formation. Curr Biol 16, 743-754

53. Sillje, H.H., et al. (2006) HURP is a Ran-importin beta-regulated protein that stabilizes kinetochore microtubules in the vicinity of chromosomes. Curr Biol 16, 731-742

54. Tulu, U.S., et al. (2003) Peripheral, non-centrosome-associated microtubules contribute to spindle formation in centrosome-containing cells. Curr Biol 13, 1894-1899

55. Carazo-Salas, R.E., et al. (2001) Ran-GTP coordinates regulation of microtubule nucleation and dynamics during mitotic-spindle assembly. Nat Cell Biol 3, 228-234

56. Kalab, P., et al. (2006) Analysis of a RanGTP-regulated gradient in mitotic somatic cells. Nature 440, 697-701

57. Kalab, P., et al. (2002) Visualization of a Ran-GTP gradient in interphase and mitotic Xenopus egg extracts. Science 295, 2452-2456

58. Nachury, M.V., et al. (2001) Importin beta is a mitotic target of the small GTPase Ran in spindle assembly. Cell 104, 95-106

59. Clarke, P.R., and Zhang, C. (2008) Spatial and temporal coordination of mitosis by Ran GTPase. Nat Rev Mol Cell Biol 9, 464-477

60. Karsenti, E., and Vernos, I. (2001) The mitotic spindle: a self-made machine. Science 294, 543-547

61. Gruss, 0.J., et al. (2001) Ran induces spindle assembly by reversing the inhibitory effect of importin alpha on TPX2 activity. Cell 104, 83-93

62. Wittmann, T., et al. (2000) TPX2, A novel xenopus MAP involved in spindle pole organization. J Cell Biol 149, 1405-1418 
63. Ma, N., et al. (2011) TPX2 regulates the localization and activity of Eg5 in the mammalian mitotic spindle. J Cell Biol 195, 87-98

64. Bayliss, R., et al. (2003) Structural basis of Aurora-A activation by TPX2 at the mitotic spindle. Mol Cell 12, 851-862

65. Eyers, P.A., et al. (2003) A novel mechanism for activation of the protein kinase Aurora A. Curr Biol 13, 691-697

66. Tsai, M.Y., et al. (2003) A Ran signalling pathway mediated by the mitotic kinase Aurora A in spindle assembly. Nat Cell Biol 5, 242-248

67. Groen, A.C., et al. (2004) XRHAMM functions in ran-dependent microtubule nucleation and pole formation during anastral spindle assembly. Curr Biol 14, 1801-1811

68. Yokoyama, H., et al. (2014) The nucleoporin MEL-28 promotes RanGTPdependent gamma-tubulin recruitment and microtubule nucleation in mitotic spindle formation. Nat Commun 5, 3270

69. Caudron, M., et al. (2005) Spatial coordination of spindle assembly by chromosome-mediated signaling gradients. Science 309, 1373-1376

70. Ribbeck, K., et al. (2006) NuSAP, a mitotic RanGTP target that stabilizes and cross-links microtubules. Mol Biol Cell 17, 2646-2660

71. Yokoyama, H., et al. (2008) Cdk11 is a RanGTP-dependent microtubule stabilization factor that regulates spindle assembly rate. J Cell Biol 180, 867-875 72. Yokoyama, H., et al. (2009) ISWI is a RanGTP-dependent MAP required for chromosome segregation. J Cell Biol 187, 813-829

73. Tseng, B.S., et al. (2010) Dual detection of chromosomes and microtubules by the chromosomal passenger complex drives spindle assembly. Dev Cell 18, $903-912$ 
74. Gadea, B.B., and Ruderman, J.V. (2006) Aurora B is required for mitotic chromatin-induced phosphorylation of 0p18/Stathmin. Proc Natl Acad Sci U S A $103,4493-4498$

75. Chan, Y.W., et al. (2012) Aurora B controls kinetochore-microtubule attachments by inhibiting Ska complex-KMN network interaction. J Cell Biol 196, $563-571$

76. Tsai, C.Y., et al. (2011) Aurora-A phosphorylates Augmin complex component Hice1 protein at an $\mathrm{N}$-terminal serine/threonine cluster to modulate its microtubule binding activity during spindle assembly. J Biol Chem 286, $30097-$ 30106

77. Zhu, H., et al. (2008) FAM29A promotes microtubule amplification via recruitment of the NEDD1-gamma-tubulin complex to the mitotic spindle. J Cell Biol 183, 835-848

78. Wainman, A., et al. (2009) A new Augmin subunit, Msd1, demonstrates the importance of mitotic spindle-templated microtubule nucleation in the absence of functioning centrosomes. Genes Dev 23, 1876-1881

79. Johmura, Y., et al. (2011) Regulation of microtubule-based microtubule nucleation by mammalian polo-like kinase 1. Proc Natl Acad Sci U S A 108, $11446-11451$

80. Liu, T., et al. (2014) Augmin triggers microtubule-dependent microtubule nucleation in interphase plant cells. Curr Biol 24, 2708-2713

81. Murata, T., et al. (2005) Microtubule-dependent microtubule nucleation based on recruitment of gamma-tubulin in higher plants. Nat Cell Biol 7, 961-968

82. Sanchez-Huertas, C., and Luders, J. (2015) The augmin connection in the geometry of microtubule networks. Curr Biol 25, R294-299 
83. Kollman, J.M., et al. (2011) Microtubule nucleation by gamma-tubulin complexes. Nat Rev Mol Cell Biol

84. Magiera, M.M., and Janke, C. (2014) Post-translational modifications of tubulin. Curr Biol 24, R351-354 


\section{Figure legend}

\section{Figure 1: Model for acentrosomal MT assembly}

A. RCC1, bound to the chromosomes, induces the concentration of RanGTP around the chromatin. The RanGTP gradient (blue) promotes the dissociation of spindle assembly factors, such as TPX2, from their inhibitory binding to importins (black). This then promotes the nucleation of acentrosomal MTs around the chromosomes, in a process dependent on TPX2 and on the $\gamma$ TuRC.

B. The chromosomal passenger complex CPC is located at the kinetochore. There, Aurora B phosphorylates and thus inactivates the MT-destabilizing factors MCAK and OP18. There is therefore a gradient of Aurora B dependent phosphorylation (blue) around the kinetochore acting as a hot-spot suitable for MT stabilization. MTs are therefore preferentially stabilized in the kinetochore area.

C. The augmin complex is recruited on the MT nucleated and stabilized through the RanGTP and the CPC pathways and on the centrosomal MTs. Augmin promotes MT branching an amplification, by a mechanism that seems to be potentiated by the RanGTP pathway.

D. Acentrosomal MT plus-ends are stabilized at the kinetochores through their interaction with the KMN complex. Minus-ends are pushed away towards the poles. MTs are organized in bundles and form the K-fiber. 
A. RanGTP-dependent MT nucleation around the chromosomes

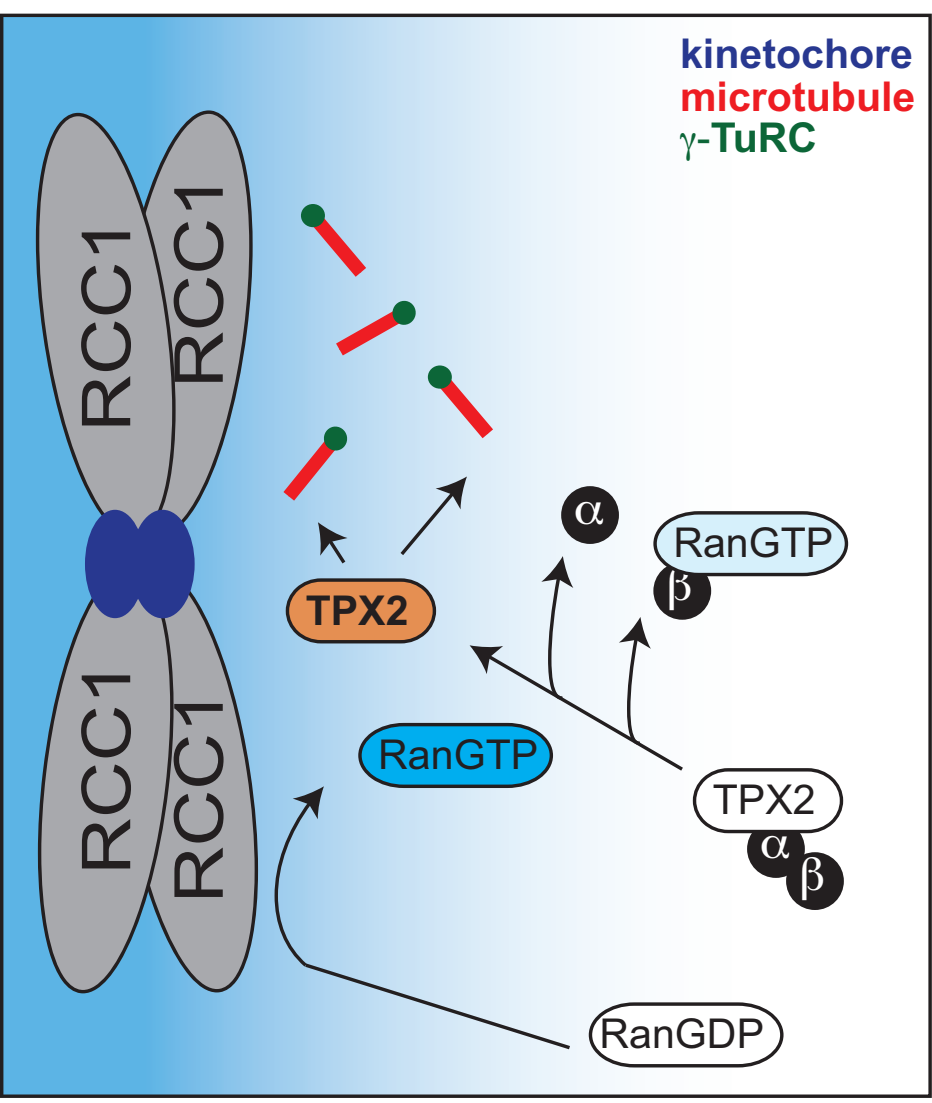

C. Augmin-dependent MT amplification on Chromosomal and centrosomal MTs

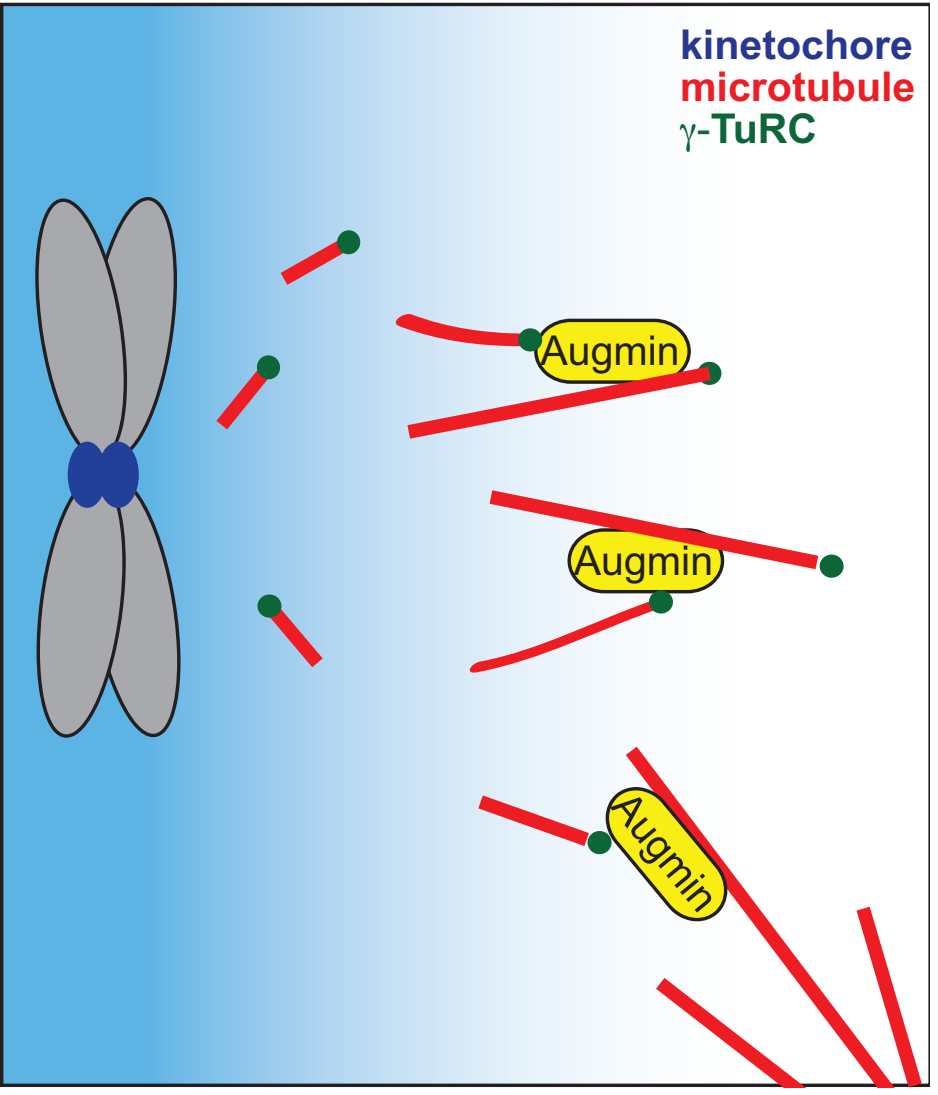

B. CPC-dependent MT stabilization around kinetochores

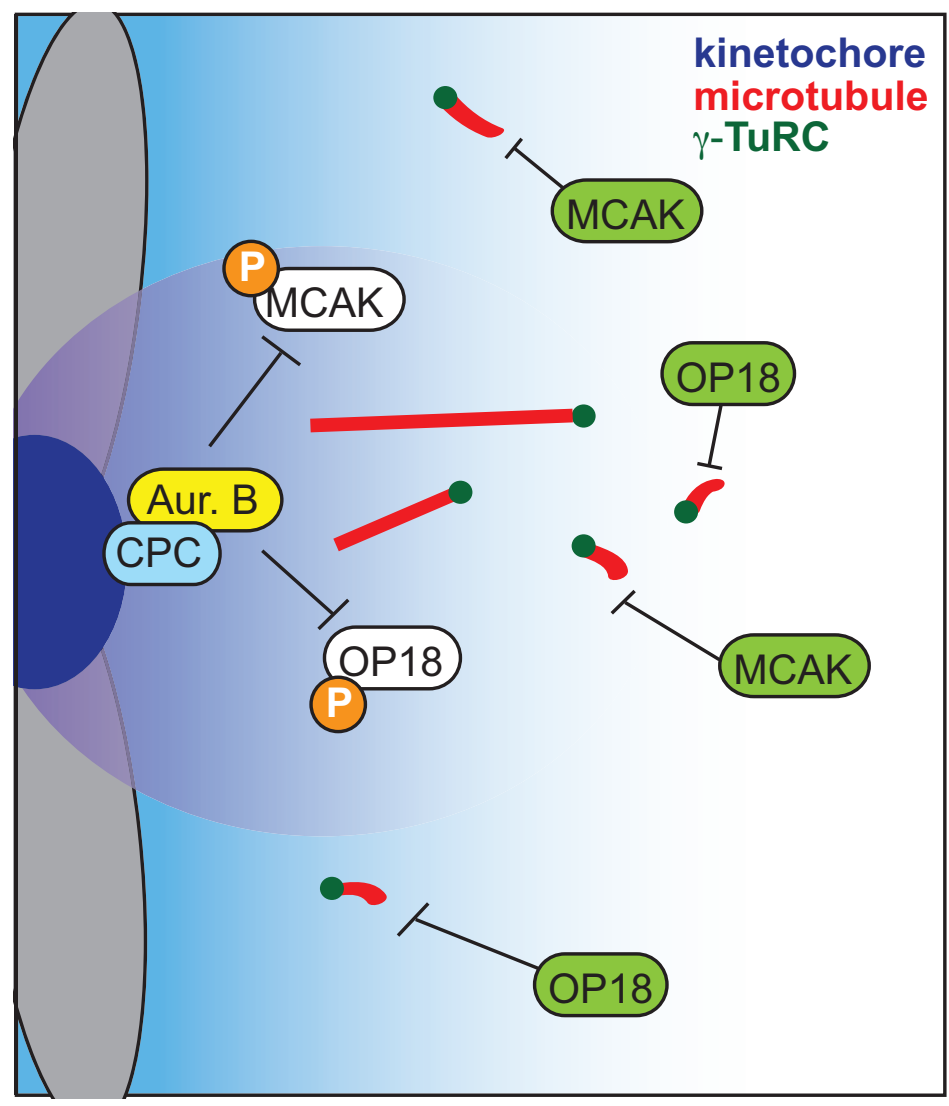

D. K-fiber formation

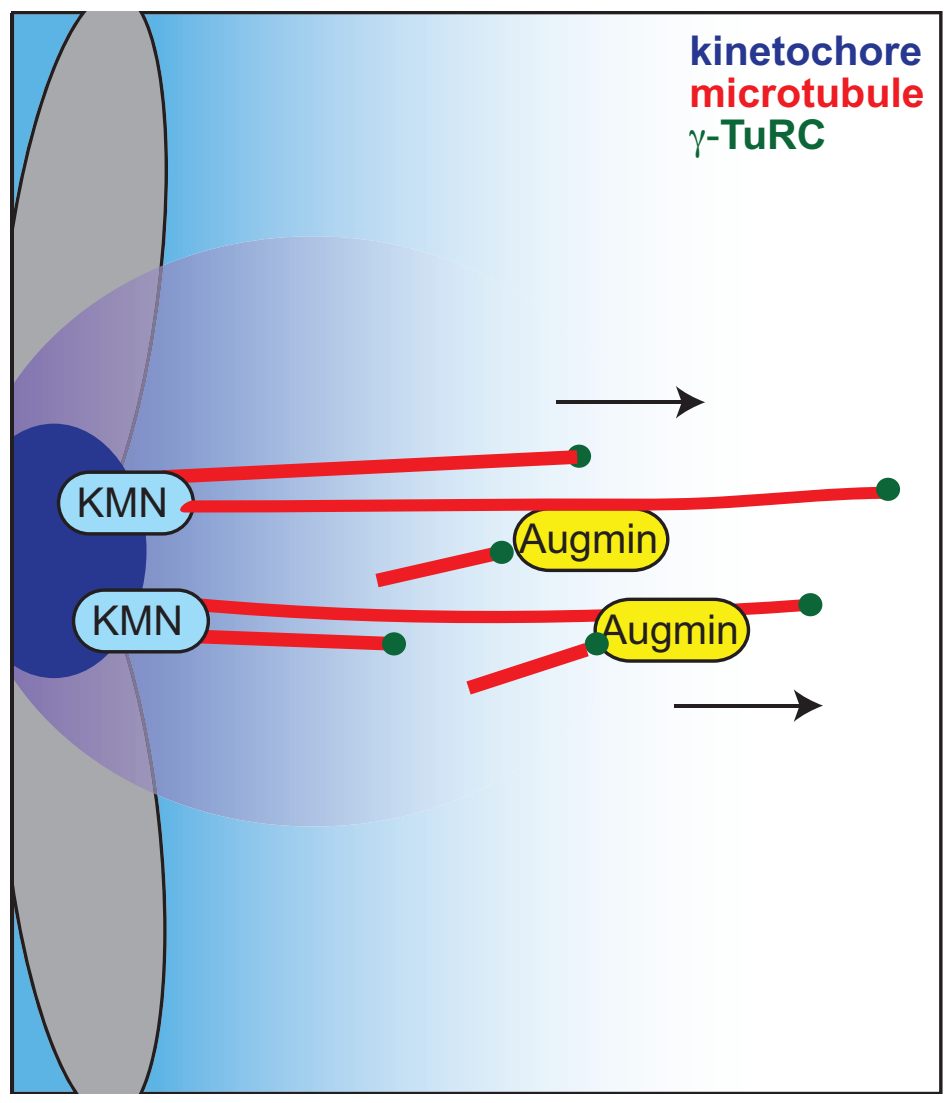




\section{Boxes}

\section{Box 1: The RanGTP pathway}

In the dividing cell, the association of the Ran-GEF RCC1 with the chromatin promotes a peak of GTP-bound Ran (RanGTP) close to the chromosomes generating a gradient 28-30,55-58. By associating with importin beta with high affinity, RanGTP promotes locally the dissociation of a number of NLS (nuclear localization signal) containing proteins called spindle assembly factors (SAFs) from importins alpha and/or beta 59,60. The released SAFs promote MT nucleation, stabilization and organization in the vicinity of the chromosomes. There are currently around 12 proteins identified as direct or indirect targets of RanGTP in the dividing cell ${ }^{3}$. One essential RanGTP target is the nuclear protein TPX2 that plays multiple roles during spindle assembly ${ }^{31,61-63}$. Once released from Importins alpha/beta by RanGTP, TPX2 interacts with the kinase Aurora A locking it in an active conformation ${ }^{64-66}$. In this way the RanGTP gradient may be translated into an Aurora A phosphorylation gradient. We recently showed that this specific activation is part of the mechanism by which RanGTP promotes MT nucleation. Indeed, RanGTP release TPX2 that interacts with a specific MT nucleation complex including the $\gamma$ TuRC, NEDD1 and RHAMM and promotes the essential phosphorylation of NEDD1 on ser405 by Aurora A, altogether driving MT nucleation $31,61,67$. Other RanGTP targets were proposed to play a role in MT nucleation 68 and stabilization around the chromatin 3,53,69-72. The RanGTP gradient is therefore translated into MT nucleation and stabilization gradients centered on the mitotic chromatin favoring the "search and capture" mechanism, the formation of the K-fibers and spindle assembly ${ }^{69}$. 


\section{Box 2: The CPC pathway}

Another mechanism was shown to promote MT assembly around the chromosomes. This pathway relies on the kinetochore-associated chromosomal passenger complex (CPC). The CPC is composed of INCENP, survivin, borealin and the Aurora-B kinase ${ }^{35}$. The role of the CPC in MT stabilization and spindle assembly has been demonstrated using the Xenopus egg extract system $32,33,73$. INCENP is an activator of the Aurora B kinase that phosphorylates and inhibits various MT destabilizing factors such as MCAK and OP18 ${ }^{74}$. The resulting Aurora-B-dependent phosphorylation gradient is postulated to favor MT assembly in the kinetochore area. However, the CPC has complex functions during mitosis as it also participates in error correction through Aurora-B dependent phosphorylation of components of the KMN network ${ }^{35}$. In this context the phosphorylation of NDc80 or of Ska complex components by Aurora B destabilizes kinetochore - MT attachments ${ }^{75}$.

The CPC-dependent and RanGTP dependent mechanisms appear to act independently to favor MT assembly. In fact it is very likely that the two systems cooperate for preferential MT assembly in the kinetochore area 36,73 .

\section{Box 3: The augmin pathway}

The augmin complex also called HAUS in mammalian systems was identified in various organisms in the last few years ${ }^{42-44}$. It interacts with and targets the $\gamma$ TURC to the side of a pre-existing MT driving MT-dependent MT nucleation ${ }^{46}$. The augmin complex consists of 8 proteins. One of them, FAM29A, binds to the $\gamma$ TuRC while another one, HICE1, directly associates with the lattice of a preexisting MT 76,77. The nucleation of a new MT on the lattice of a pre-existing one 
results in MT branching 45,46 . The branched MTs are then transported along the pre-existing MTs towards the spindle poles ${ }^{50}$. The augmin complex can in principle associate to any MT and in mitosis it acts on centrosomal and acentrosomal MTs 43, 49 .

By exponentially amplifying the number of MTs in mitosis, the augmin dependent pathway constitutes a very efficient system for MT amplification 47, 49 that contributes to the robustness of the mitotic spindle. However, the contribution of the augmin-dependent MT assembly pathway to spindle assembly is probably different depending on the systems $43,47,78$.

The augmin pathway is highly regulated by mitotic kinases such as Aurora A and PLK1, that are necessary for its activity in mitosis 76,79 .

In contrast to the RanGTP and the CPC-dependent MT assembly machineries that are nuclear in interphase and therefore can only act after nuclear envelope breakdown, the augmin complex may also play a role in MT nucleation in interphase, as this activity has been reported in plants ${ }^{80-82}$. 


\section{Outstanding questions}

- Both the $\gamma$ TuRC and its associated protein NEDD1 are required for the three different MT nucleation pathways described here. Interesting, each pathway involves NEDD1 phosphorylation, but through different kinases (cdk1, Nek9 and Aurora A), each targeting a single and distinct residue albeit all in the same region of the protein. How NEDD1 phosphorylation is translated into $\gamma$ TuRC activation and MT nucleation is however still unclear. The understanding of the activation of MT nucleation in mitosis may therefore require a better understanding of NEDD1 function and regulation ${ }^{83}$.

- The contribution of each MT assembly pathway in the composition of the spindle differs greatly depending on the organisms, cell types, and in mitotic versus meiotic systems. Some outstanding questions are: How are these different pathways integrated to build the mitotic spindle? What is the qualitative contribution of each pathway to the function of the spindle, if any? Although we have discussed here the possibility that chromosomal MTs have an essential role in K-fiber formation, there is currently no direct evidence to support this hypothesis. Further work should explore this idea.

- The augmin-dependent pathway and its role in MT-dependent MT nucleation and amplification has only been recently described. Some important questions should still be addressed to fully understand how it works. While the function of the augmin complex in animal cells has only been described during mitosis, in plants, it was also shown to function in interphase ${ }^{80,82}$. Is the augmin dependent 
pathway active throughout the cell cycle in animal cells or is it restricted to mitosis like the RanGTP-dependent pathway?

- It was recently described that augmin-dependent MT nucleation sites are transported toward the spindle poles along pre-existing MTs in metaphase 50. Does the augmin complex bind to specific predefined sites or randomly along the MT lattice? MT post-translational modifications can alter the MT binding activity of a number of MT associated proteins (MAPs) ${ }^{84}$. Could a related mechanism define sites for augmin-binding, and therefore where MT branching occurs within the mitotic spindle?

- The mechanism by which the augmin-dependent MT nucleation only occurs when bound to a pre-existing MT is unclear. Is there a sequential recruitment of components to the MT lattice? Does the augmin complex bind first to the MT lattice and then recruits the $\gamma \mathrm{TuRC}$, or are there preformed inactive augmin$\gamma$ TuRC complexes in the cytoplasm? Work with in vitro reconstituted systems ${ }^{44}$ may provide the answers to these key questions.

- A recent work suggested a putative functional link between TPX2 and the augmin complex ${ }^{45}$. In the light of the recent identification of a specific MT nucleation complex for the RanGTP-dependent pathway (RHAMM-NEDD1$\gamma$ TuRC) ${ }^{31}$, it will be important to determine if this complex is also used by the augmin pathway to nucleate MTs. More generally, are there different specific $\gamma$ TuRCs subcomplexes that are 'primed' for activation through each of the Mphase MT assembly pathways? 
- Are the MTs nucleated through the distinct pathways functionally identical? There is currently only one example for the specific association of a protein to only one class of acentrosomal MTs ${ }^{41}$. The identification of other specific acentrosomal MT binding partners if they exist, could fuel our understanding on the function of these MTs during mitosis. 